\title{
La representación cognitiva de los verbos. Aproximación descriptiva y evolutiva
}

\author{
ISABELLE BONNOTTE \\ ANTON KAIFER \\ MICHEL FAYOL \\ ITZIAR IDIAZÁBAL \\ Université de Bourgogne y Universidad del Pais Vasco \\ Resumen
}

Con el objeto de tener un mayor conocimiento del funcionamiento de las formas verbales en castellano, este trabajo aporta datos empiricos relativos a la manera cómo se representan las características del proceso (Aktionsart) descritas por el verbo. Tres son los factores estudiados: la duratividad, la resultatividad y la iteratividad o continuidad del proceso. Utilizando una metodología de representación gráfica se han recogido datos de una población de 42 adultos $y$ 72 niños distribuidos en 4 grupos correspondientes a los cursos $2 .^{\circ}, 4 .^{\circ}, 6 .^{\circ}$ y 8.

Los resultados obtenidos con los nueve verbos estudiados demuestran, por un lado, la validez del método ya que bace aparecer una neta dicotomia que opone los procesos resultativos no durativos a los no resultativos durativos, $y$ por otro, que la duratividad y la resultatividad del proceso son captados de manera muy semejante por los adultos $y$ por los niños de $4 .^{\circ}, 6 .^{\circ} y$ $80^{\circ}$, si bien los de $2 .^{\circ}$ denotan un menor acuerdo interindividual. Por último, el factor duratividad o iteratividad no constituye en esta prueba una dimensión discriminante.

Palabras clave: Representación cognitiva, proceso del verbo, aktionsart, resultatividad, duratividad, iteratividad, continuidad.

\section{Cognitive representation of verbs: A descriptive and developmental approach}

\section{Abstract}

With the object of increasing our present knowledge of verbal forms in Spanish, this study provides empirical data on bow the characteristics of the process (or Aktionsart) described by the verb are represented. Three characteristics were studied: durativity, resultativity, and iterativity or continuity of the process. A methodology of graphic representation was used in the data gathering procedure on a population of 42 bistory undergraduates and 72 children, distributed in four age groups with a mean age of $7,9,11$, and 13 years. The results from the nine verbs studied show: a) the method's validity in providing a clear dichotomy between resultive nondurative methods and non-resultive durative methods; $b$ ) the durativity and resultivity of the processes studied are grasped in a very similar manner by both adults and children in the 9 , 11 , and 13 age groups, though the youngest children -7 years- show lower interindividual agreement; finally, $c)$ in this study durative or iterative factors do not represent a discriminative dimension.

Keywords: Cognitive representation, verb process, Aktionsart, resultivity, durativity, iterativity, continuity.

Agradecimientos: Esta investigación ha sido realizada dentro del programa de acciones integradas hispano-francesas n. 66 de 1990 . Agradecemos a Idioa Irnaz y Pilar Larrañaga su colaboración en la recogida del material de este trabajo.

Dirección de las autoras: Université de Bourgogne L.E.A.D. 36, rue Chabot-Charny 21000 Dijon (Francia). Universidad del País Vasco. Facultad de Filologia. Marqués de Urquijo, s/n. (01006 Vitoria).

Original recibido: Mayo 1990. Revision recibida: Noviembre 1990. Aceptado: Noviembre 1990. 


\section{SITUACION DEL PROBLEMA}

En el estudio del desarrollo ontogenético del uso de las formas verbales aparecen, en castellano y en otras lenguas, una serie de problemas. Se observan, en efecto, más o menos simultáneamente, cambios que afectan al conjunto de formas disponibles, a su frecuencia relativa y a su funcionamiento. Así,

- Szagun (1978), en inglés y en alemán, Malrieu (1976), Malrieu y Laterasse (1976), para el francés y Gili Gaya (1972) y Hernández Pina (1984), para el español, observan patrones de empleo muy parecidos en los niños: en todas estas lenguas, el presente parece ser la primera forma conjugada, seguida del pasado y después del futuro, aumentando paulatinamente el número de formas disponibles;

- El conjunto de autores que trabajan sobre el desarrollo observan que la aparición de una nueva forma verbal supone una modificación, más o menos profunda, de la distribución de frecuencias relativas respecto de las anteriormente utilizadas;

- Varios autores han observado reorganizaciones en los procedimientos de utilización de las formas verbales. Por ejemplo, Antinucci y Miller (1976) destacan que el pretérito (que corresponde en castellano al imperfecto) solamente se aplica inicialmente (2,1 años) a las descripciones de estado o de procesos no-resultativos. Las observaciones de Bronckart (1976) demuestran, también, cambios en el "valor" de las formas verbales del pasado, en francés (Cf. Fayol, 1983, para el pluscuamperfecto en francés).

Estas modificaciones observables en los comportamientos lingüísticos conducen a preguntarse por qué se producen: ¿Qué es lo que cambia? ¿Qué es lo que se desarrolla?

Para intentar responder a estas cuestiones conviene plantear algunas hipótesis, en relación con los diferentes parámetros actualmente considerados como responsables de la regulación del uso de las formas verbales. Sin duda, el conocimiento más exacto de los factores que influyen en el uso de las diferentes formas verbales puede contribuir a mejorar la comprensión/producción en una lengua $y$, a partir de ello, a desarrollar estrategias didácticas que permitan un aprendizaje más preciso en los diferentes niveles del desarrollo, es decir, en cada grupo de nivel o de edad.

En un primer análisis vamos a considerar tres categorías de factores intervinientes:

a) La construcción del referente en relación al que se encuentran situados los estados a los acontecimientos. De acuerdo con Reichenbach (1947), se puede considerar que los estados o acontecimientos evocados tienen una primera referencia en el momento de enunciación —de ahí la posibilidad de construir el pasado/presente/futuro- pero también tiene un referente derivado: el punto de vista adoptado por el locutor ante el estado o el proceso. Este doble sistema de referencia posibilita el «cálculo» complejo que implica la "elección» de algunos tiempos (pluscuamperfecto, imperfecto y futuro anterior). Desde esta perspectiva, parece posible plantear un desarrollo ontogenético en dos fases: la primera referida a la instauración del cálculo relativo al momento de enunciación; la segunda integra en 
este cálculo el que corresponde al punto de vista. Es ésta, resumidamente, la concepción defendida por Weist (1984); concepción que se encuentra reforzada por resultados empíricos no directamente sobre las mismas formas verbales, sino sobre ciertos ítems lexicales (Cf. Harner, 1982). Así, en francés, mientras que los empleos de «aujourd'hui (hoy)/hier (ayer)/demain (mañana)" son relativamente precoces (hacia 3 ó 4 años), las apariciones correctas de "la veille (la víspera)/le lendemain (al día siguiente)» son mucho más tardías (¿8-9 años?). Parece posible, pues, postular la construcción sucesiva de estos dosreferentes como uno de los determinantes de la evolución de las formas verbales. Sin embargo, esto no es suficiente.

b) La adquisición de paradigmas lingüisticos asociados a cada «tiempon constituye otro determinante muy a menudo ignorado. Así, es necesario, en un momento o en otro, que el niño adquiera las formas y no puede hacerlo más que en contacto con un corpus que le proporcione una base de inducción, en la que inevitablemente algunas formas son más frecuentes y/o más fáciles de realizar (fonológicamente, después, ortográficamente) que otras. En consecuencia, la adquisición y el empleo de estos paradigmas dependen muy estrechamente del entorno lingüístico del niño. Pero tampoco este determinante es suficiente.

c) A la construcción, sin duda tardía, de un doble sistema de referentes y a la adquisición de un paradigma relativamente amplio y complejo de las formas verbales, se añade la necesidad de tener en cuenta, al menos para las formas del pasado, las características del proceso descrito por el verbo (Aktionsart). En efecto, como desde hace tiempo lo han subrayado los lingüistas y evidenciado empíricamente en el caso del francés Fayol, Abdi y Gombert (1989) y Fayol, Gombert y Abdi (1988), existen muy fuertes afinidades entre algunas formas verbales del pasado y algunos tipos de procesos. Estas afinidades se traducen en asociaciones frecuentes entre, por ejemplo, el imperfecto y los verbos de tipo «jouer» (jugar», danser (danzar)..." y entre el "passé simple». (perfecto simple) y los verbos «tomber (caer), exploser (explotar)...». Se sabe, también, que estas asociaciones se manifiestan en los niños. Así, Bronckart (1976), Mapstone y Harris (1985), Smith (1980) - por referirnos a algunos autores- han evidenciado claramente que los niños de 4 a 6 años son sensibles a las características temporales de las acciones descritas y asocian a ellas, de manera bastante regular, algunas formas verbales. Sin embargo, estas asociaciones diferentes, que aparecen en los niños, no tienen el mismo carácter sistemático que en los adultos (intra e inter-individual).

Si nos situamos en términos evolutivos, aparece claramente que existe a la vez un desarrollo, sin duda cognitivamente determinado, relativo a la construcción de los referentes (momento de enunciación, primero, y después, punto de vista) y una adquisición de paradigmas de realización formal, muy dependiente, sin duda, del corpus al que el niño está confrontado. Sin embargo, en el estado actual de nuestros conocimientos, se ignora casi todo sobre las eventuales modificaciones de las representaciones cognitivas de los tipos de procesos según la edad. Como hemos observado en adultos de lengua francesa, estas representaciones tienen un papel más importante en la selección de las formas verbales. Es posible, pues, que una parte de los «errores» apreciados en los niños en el uso de formas verbales, en diferentes niveles del desarrollo, tenga relación con el hecho de que esas re- 


\section{4}

presentaciones cognitivas no están, en el caso de estos niños, organizadas como en los adultos. En consecuencia, a las dificultadese relacionadas con la construcción cognitiva de referentes y a la organización de los sistemas flexionales, se añadiría el problema de la elaboración y, más adelante, de la estabilización de las representaciones cognitivas de los procesos.

Dicho de otra forma, el problema de la evolución del uso de las formas verbales nos conduce a proponer, al menos, tres hipótesis. Las variaciones observadas en las producciones de individuos de edades diferentes en lo que a los tiempos se refiere, pueden ser explicadas por:

1. Una variación en las funciones atribuidas a los diferentes tiempos verbales.

2. Una variación que afecta a las representaciones cognitivas de los procesos descritos por los verbos. Podemos preguntarnos si algunos procesos no resultarian más fácilmente aprehendibles que otros, para los niños, y si esto no produciría, en consecuencia, representaciones cognitivas más precozmente estabilizadas. Sin embargo, el problema puede plantearse de oira forma; en efecto, podemos preguntarnos si la aparición simultánea de ciertos tipos de verbos y ciertas formas verbales no conduciría a estabilizar las representaciones cognitivas de los procesos asociadas a esos verbos.

3. Una variación simultánea de las funciones atribuidas a las diferentes formas verbales y de las representaciones cognitivas de los procesos descritos por los verbos.

El objetivo del presente trabajo es precisamente proporcionar una contribución esencialmente descriptiva al estudio de una parte de este problema. En efecto, se trata aquí de comparar algunas de las dimensiones significativas de la representación cognitiva (carácter durativo o no, resultativo o no, iterativo o semelfactivo) de los mismos procesos, tal como es elaborada por el adulto y el niño en los diferentes niveles de su desarrollo. Debemos intentar acceder a estas representaciones utilizando métodos que no impliquen $\multimap$ lo hagan en la menor medida posible-el recurso al lenguaje.

De acuerdo con este objetivo, hemos recurrido a una tarea de representación gráfica de las dimensiones anteriormente mencionadas (carácter durativo o no, resultativo o no, iterativo o no). Esta tarea tiene la ventaja de ser fácil de realizar -incluso para los niños más jóvenes (6-7 años) - y de ser bien aceptada. Presenta poco riesgo de "bloqueo» o de subestimación, pues al sujeto no se le pide ni que se exprese verbalmente, ni que dé una opinión. el sujeto debe simplemente trazar, sobre una hoja preparada para ello, líneas más o menos largas, continuas o no y con su extremo claramente marcado o no, para así codificar la mayor o menor duratividad, iteratividad y resultatividad en los procesos descritos por los verbos que se le presentan en infinitivo.

\section{METODO}

\subsection{Población}

Hemos propuesto la prueba a 42 estudiantes inscritos en $4 .^{\circ}$ curso de Historia de la Universidad del País Vasco y a 72 niños de una escuela de 
Vitoria, pertenecientes a $2 .^{\circ}, 4 .^{\circ}, 6 .^{\circ}$ y $8 .^{\circ}$ curso de EGB $(18$ nin̄os por curso). Todos los sujetos son de lengua materna castellana.

Para el grupo "niños", indicamos en la Tabla I las edades medias y las edades límites por curso, así como los números de alumnos en función del sexo.

\section{TABLA I}

Edades medias y limites por curso y número de alumnos de la población infantil según el sexo

\begin{tabular}{lcccc}
\hline & Edad media & Edad límite & N. ${ }^{\circ}$ niños & N..$^{\circ}$ iñas \\
\hline 2. & & & & \\
4. EGB & 7,3 & $7-7,9$ & 10 & 8 \\
6. EGB & 9,1 & $8,11-9,4$ & 9 & 9 \\
8. ${ }^{\circ}$ EGB & 11 & $10,9-11,2$ & 9 & 9 \\
\hline
\end{tabular}

\subsection{Material}

Hemos tenido en cuenta tres categorias verbales que, a priori, corresponden aproximadamente a las categorias de Vendler (1967):

1. (AR) Categoría de verbos arresultativos (andar, jugar, danzar), correspondientes a expresiones de actividad («activity terms» para Vendler).

2. (RD) Categoría de verbos resultativos durativos (avanzar, subir, preparar), correspondientes a expresiones de cumplimiento («accomplishment terms» para Vendler).

3. (RI) Categoría de verbos resultativos instantáneos (volcar, explotar, romper), correspondientes a expresiones de acabamiento (*achievement terms» para Vendler).

\subsection{Procedimiento}

Los sujetos deben realizar juicios sobre 16 ítems verbales: los 9 ítems verbales analizados y 7 ítems verbales utilizados como "distractores» que no se analizan en esta experiencia. Estos ítems pueden ser considerados como pertenecientes a las categorias (E) de estado e (IT) iterativa. A priori, los sujetos pueden, pues, representar el conjunto de los ítems (E, AR, IT, $\mathrm{RD}, \mathrm{RI}$ ) utilizando todas las definiciones propuestas (E, AR, IT, RD, RI). Por otro lado, hemos empleado cuatro formas de presentación de los 16 ítems verbales, ordenados de forma distinta en cada una de ellas, para evitar un posible efecto de listas (cuatro listas diferentes). La posición de cada ítem en cada lista ha sido atribuida al azar.

El objetivo que pretendemos lograr es el de transmitir un cierto número de informaciones que sean correctamente utilizadas por el sujeto. Así pues, hemos controlado sistemáticamente que los niños lleguen a dominar el código gráfico y lo utilicen correctamente. Este control nos ha parecido necesario en el caso de los niños debido al carácter abstracto de las informaciones que se les transmite. 


\subsubsection{Procedimiento utilizado con los adultos}

\subsubsection{Fase de aprendizaje}

En una primera fase el experimentador transmite al sujeto la información que le va a permitir, después, la representación gráfica de los ítems verbales. Distribuye a cada sujeto una hoja de las consignas y las lee para asegurarse que los sujetos comprenden bien lo que se espera de ellos. No utiliza ningún ejemplo para evitar que los sujetos procedan por imitación durante la prueba (responder asociando cada ítem verbal analizado al ítem verbal utilizado durante la sesión de aprendizaje).

1. Los verbos de estado $(E)$. Consigna: "Cuando encuentres verbos que sirven para relatar situaciones y que no evocan una acción del sujeto, debes hacer un trazo horizontal comenzando por el extremo izquierdo de la hoja hasta llegar al extremo derecho».

FIGURA 1

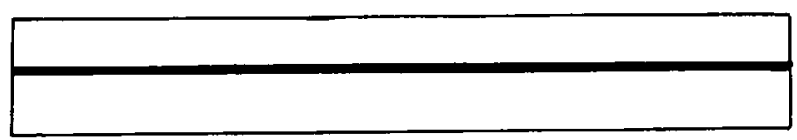

2. Los verbos arresultativos (AR). Consigna: «Cuando encuentres verbos que evocan situaciones que duran mucho tiempo y no dan lugar a un resultado directamente observable (el resultado de la acción solamente puede ser inferido), deberás hacer un trazo comenzando por el extremo izquierdo de la hoja pero que no llegue hasta el derecho. Este trazo deberá ser tan largo como la duración de la acción».

\section{FIGURA 2}

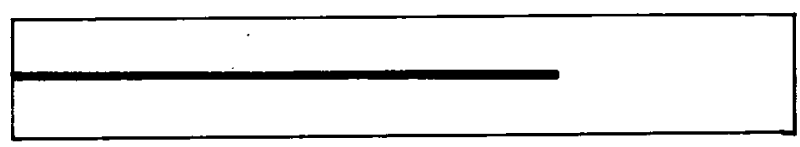

3. Los verbos iterativos (IT). Consigna: «Cuando encuentres verbos que evocan una actividad compuesta por varias pequeñas actividades idénticas y que no den lugar a un resultado directamente observable (el resultado de la acción solamente puede ser inferido), deberás hacer un trazo discontinuo, compuesto por guiones, partiendo siempre del extremo izquierdo de la hoja, pero sin llegar hasta el derecho. Este trazo deberá ser tan largo como la duración de la acción». 
4. Los verbos resultativo durativos $(R D)$ o instantáneos ( $R I)$. Consigna: «Cuando encuentres verbos que describen situaciones de duración variable y que claramente dan lugar a un resultado directamente observable, deberás hacer un trazo comenzando por el extremo izquierdo de la hoja, pero sin llegar al extremo derecho. Este trazo deberá ser tan largo como la duración de la acción. Deberás añadir un pequeño trazo vertical que representa una acción que da lugar a un resultado directamente observable».

FIGURA 4

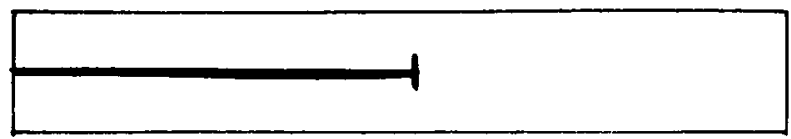

\subsubsection{Fase test}

El experimentador entrega a los sujetos una hoja en la que están impresos los 16 ítems verbales y se les pide que representen gráficamente cada uno de ellos, utilizando el código aprendido durante la fase precedente (cada sujeto dispone de una hoja con las consignas durante toda la prueba).

\subsubsection{Procedimiento utilizado con los niños}

\subsubsection{Fase de aprendizaje}

El aprendizaje del código gráfico se desarrolla en dos sesiones (colectivas en cada curso; duración aproximada, media hora). El experimentador se refiere a verbos diferentes a los analizados, pero pertenecientes a las mismas categorías. El recurso a ejemplos para que los niños aprendan el código gráfico nos ha parecido indispensable por el carácter abstracto de este código y por las edades de los niños (en 2..$^{\circ}$ de EGB los más jóvenes). A continuación señalamos la lista de los verbos empleados durante las dos sesiones de aprendizaje:

a) Verbos de estado: habitar, poseer, brillar.

b) Verbos arresultativos: arrastrarse, chupar.

c) Verbos iterativos: acariciar, saltar (a la cuerda), golpetear.

d) Verbos resultativos durativos: dibujar, vestirse, desvestirse, distribuir, extender.

e) Verbos resultativos instantáneos: cerrar, esconder, encender.

Durante la primera sesión, el experimentador integra un verbo en una frase y ejecuta la acción, muestra a los niños cómo se puede codificar el verbo con la ayuda de un trazo.

1. Los verbos de estado: "Yo tengo una sortija. Esto dura todo el timpo, yo la tengo todo el tiempo. No pasa nada. Por eso hago una raya que va desde un lado de la pizarra hasta el otro." El experimentador repite varias veces e insiste en la estabilidad del proceso: «esto dura todo el tiempo".

2. Los verbos arresultativos: "Yo me chupo el dedo. Esto dura bastante tiempo y no pasa nada. Yo estoy bastante tiempo chupando, pero esto 
no dura tanto como tener. Entonces hago una raya bastante larga en la pizarra, pero que no llega hasta el final.»

3. Los verbos iterativos: "Cuando yo acaricio una muñeca bago varias veces la misma cosa, hago varias rayas cortitas, y como esto no dura todo el tiempo, me paro antes de llegar al final de la pizarra.»

4. Los verbos resultativos durativos: "Cuando dibujo una flor paso algo de tiempo entonces hago una raya bastante larga. Pero además ha pasado algo, ahora hay una flor en la pizarra. Para mostrar que ha pasado algo, voy a añadir una pequeña barra al final de la raya.»

5. Los verbos resultativos instantáneos: "Cuando cierro la ventana lo hago rápido y además pasa algo; ahora la ventana está cerrada. Para mostrar que esto se hace rápido hago una pequeña raya y para mostrar que pasa algo añado una barra al final de la raya.»

Al final de la primera sesión, el experimentador invita a los niños a ejecutar ellos mismos las acciones y a representarlas gráficamente en la pizarra. La segunda sesión se desarrolla al día siguiente. Debe permitir consolidar los conocimientos adquiridos la víspera. Según las indicaciones del experimentador, los niños sistemáticamente han imitado y representado gráficamente en la pizarra los ejemplos vistos en la sesión precedente. Sus representaciones gráficas correspondían al código transmitido.

\subsubsection{Fase test}

Los niños de $2 .^{\circ}, 4 .^{\circ}$ y $6 .^{\circ}$ curso han realizado la prueba en grupos de cinco aproximadamente ocho días después de la segunda sesión de aprendizaje. Los niños de $8 .^{\circ}$ curso han realizado la prueba colectivamente en el mismo espacio de tiempo. Este intervalo de tiempo está destinado a evitar que los verbos sean directamente asimilados a los utilizados durante la fase de entrenamiento. Antes del comienzo de la prueba, el experimentador se asegura que los niños han dominado bien el código gráfico, lo que se ha producido en todos los casos. A continuación, el experimentador proporciona a los niños una hoja en la que están impresos los 16 ítems verbales y les pide que representen cada ítem verbal, utilizando el código aprendido.

\section{RESULTADOS}

Con el fin de facilitar el tratamiento, los datos han sido codificados de forma binaria. Hemos codificado, pues, para cada sujeto, cada uno de los verbos como: resultativo (RO) o no (RN); durativo (DO) o no (DN); continuativo $(\mathrm{CO})$ o no $(\mathrm{CN})$.

A partir de esta primera codificación, hemos procedido a dos tipos de tratamiento. En primer lugar, hemos analizado, para cada una de las tres dimensiones, los porcentajes relativos las caracteristicas de cada verbo y hemos calculado las correlaciones entre los porcentajes y todos los grupos. De esta manera, se trata de estudiar si las representaciones cognitivas presentan en cada una de las dimensiones, las similitudes o diferencias de un grupo a otro. A continuación, hemos efectuado, grupo por grupo, una serie de análisis factoriales de correspondencias (AFC). Este tipo de análisis, ya utilizado en un trabajo anterior (Fayol et al., 1988), permite investigar 
si, considerando simultáneamente las tres dimensiones estudiadas (duratividad, resultatividad, continuidad), se obtienen, en todos los grupos reagrupamientos similares de los ítems verbales; reagrupamientos que traducirían, entonces, una estabilidad de la manera por la que los sujetos se representan los tipos de procesos.

\subsection{Análisis por dimensión}

Los porcentajes de duratividad, resultatividad y continuidad aparecen en las Tablas II, III y IV; y los resultados de los cálculos de correlación para cada una de las dimensiones, en las Tablas V, VI y VII.

\section{TABLA II}

Porcentajes de duratividad por verbo y por grupo de edad

\begin{tabular}{lccccc}
\hline \multicolumn{1}{c}{ Verbos } & $2 .^{\circ}$ EGB & 4. $^{\circ}$ EGB & $6^{\circ}$ EGB & 8. ${ }^{\circ}$ EGB & Adultos \\
\hline ANDAR & .944 & .833 & 1 & 1 & .976 \\
JUGAR & .889 & .833 & .889 & .944 & .952 \\
DANZAR & .50 & .778 & .778 & 1 & .928 \\
AVANZAR & .889 & .889 & .889 & .833 & .952 \\
SUBIR & .722 & .667 & .50 & 1 & .905 \\
PREPARAR & .778 & .667 & .611 & .888 & .928 \\
TIRAR & .555 & .389 & .167 & .111 & .262 \\
EXPLOTAR & .555 & .278 & .167 & .389 & .19 \\
ROMPER & .50 & .278 & .167 & .333 & .19 \\
\hline
\end{tabular}

TABLA III

Porcentajes de resultatividad por verbo y por grupo de edad

\begin{tabular}{|c|c|c|c|c|c|}
\hline Verbos & 2..$^{\circ} \mathrm{EGB}$ & $4 .^{\circ} \mathrm{EGB}$ & $60^{\circ} \mathrm{EGB}$ & 8. ${ }^{\circ}$ EGB & Adultos \\
\hline ANDAR & .167 & .222 & .055 & .055 & .143 \\
\hline JUGAR & .333 & .278 & .167 & .111 & .143 \\
\hline DANZAR & .389 & .222 & .222 & .055 & .119 \\
\hline AVANZAR & .333 & .611 & .222 & .167 & .286 \\
\hline SUBIR & .389 & .389 & .333 & .055 & .286 \\
\hline PREPARAR & .555 & .444 & .555 & .055 & .809 \\
\hline TIRAR & .50 & .778 & .944 & .944 & .833 \\
\hline EXPLOTAR & .667 & .944 & .944 & .889 & .88 \\
\hline ROMPER & .778 & 1 & .944 & .944 & .928 \\
\hline
\end{tabular}

\section{TABLA IV}

Porcentajes de continuidad por verbo y por grupo de edad

\begin{tabular}{|c|c|c|c|c|c|}
\hline Verbos & $2 .^{\circ} \mathrm{EGB}$ & $40^{\circ} \mathrm{EGB}$ & $6 .^{\circ} \mathrm{EGB}$ & $8 .^{\circ} \mathrm{EGB}$ & Adultos \\
\hline ANDAR & .667 & .444 & .278 & .389 & .428 \\
\hline JUGAR & 1 & .778 & .944 & .667 & .548 \\
\hline DANZAR & .889 & .50 & .722 & .222 & .309 \\
\hline AVANZAR & .889 & .833 & .778 & .778 & .619 \\
\hline SUBIR & .944 & .778 & .778 & .444 & .595 \\
\hline PREPARAR & 1 & .889 & 1 & .889 & .786 \\
\hline TIRAR & .944 & 1 & $i$ & .944 & .833 \\
\hline EXPLOTAR & 1 & $i$ & 1 & 1 & .857 \\
\hline ROMPER & 1 & 1 & 1 & 1 & .905 \\
\hline
\end{tabular}


TABLA V

Coeficientes de correlación entre grupos calculados a partir de porcentajes de duratividad

\begin{tabular}{lccccc}
\hline & $2 .^{\circ}$ EGB & $4 .^{\circ}$ EGB & $6 .^{\circ}$ EGB & 8. ${ }^{\circ}$ EGB & Adultos \\
\hline $2 .^{\circ}$ EGB & 1 & & & & \\
$4 .^{\circ}$ EGB & $.758 *$ & 1 & & & \\
$6 .{ }^{\circ}$ EGB & $.781 *$ & $.969 * *$ & 1 & & \\
$8.0^{\circ}$ EGB & .620 & $.870 * *$ & $.859 * *$ & 1 & \\
Adultos & $.722 *$ & $.963^{* *}$ & $.918^{* *}$ & $.948 * *$ & 1 \\
\hline
\end{tabular}

$* * p<.01$

$* \mathrm{p}<.05$

TABLA VI

Coeficientes de correlación entre grupos calculados a partir de porcentajes de resultatividad

\begin{tabular}{|c|c|c|c|c|c|}
\hline & $20^{\circ} \mathrm{EGB}$ & $4 .^{\circ} \mathrm{EGB}$ & $6 .^{\circ} \mathrm{EGB}$ & 8..$^{\circ} \mathrm{EGB}$ & Adultos \\
\hline $\begin{array}{l}20^{\circ} \text { EGB } \\
40^{\circ} \text { EGB } \\
6.0^{\circ} \text { EGB } \\
8.0^{\circ} \text { EGB } \\
\text { Adultos }\end{array}$ & $\begin{array}{c}1 \\
.854 * \% \\
.895 * \% \\
.752 \approx * \\
.885 \approx\end{array}$ & $\begin{array}{c}1 \\
.901 * * \\
.915 * * \\
.856 *\end{array}$ & $\begin{array}{c}1 \\
.919 * * \\
.950 * *\end{array}$ & $\begin{array}{c}1 \\
.802\end{array}$ & 1 \\
\hline
\end{tabular}

${ }^{* *} \mathrm{P}<<.01$

* $\mathrm{p}<.05$

TABLA VII

Coeficientes de correlación entre grupos calculados a partir de porcentajes de continuidad

\begin{tabular}{|c|c|c|c|c|c|}
\hline & $20^{\circ} \mathrm{EGB}$ & $4 .^{\circ} \mathrm{EGB}$ & $6 .^{\circ} \mathrm{EGB}$ & 8. ${ }^{\circ}$ EGB & Adultos \\
\hline $\begin{array}{l}20^{\circ} \text { EGB } \\
4 .^{\circ} \text { EGB } \\
6.0^{\circ} \text { EGB } \\
80^{\circ} \text { EGB } \\
\text { Adultos }\end{array}$ & $\begin{array}{c}1 \\
.782 * \\
.967 * * \\
.606 \\
.623\end{array}$ & $\begin{array}{c}1 \\
.868 * * \\
.929 \approx * \\
.946 * *\end{array}$ & $\begin{array}{c}1 \\
.734 \\
.728\end{array}$ & $\begin{array}{c}1 \\
.953 \approx *\end{array}$ & 1 \\
\hline
\end{tabular}

$4 * \mathrm{p}<.01$

* $\mathrm{p}<.05$

El análisis de esta primera serie de resultados permite observar algunos fenómenos que vamos a abordar en cada una de las dimensiones.

\subsubsection{La duratividad}

Como revelan los resultados de la Tabla II, los adultos y los niños de 4. ${ }^{\circ}, 6 .^{\circ}$ y $8 .^{\circ}$ representan unos verbos como clara y masivamente durativos (andar, jugar, danzar, avanzar, subir, preparar) y otros como no durativos (volcar, explotar, romper). Debemos subrayar las variaciones interindividuales que afectan a «subir» y "preparar» en el grupo de $6 .^{\circ}$

Sin embargo, el consenso dentro del grupo de $2 .^{\circ}$ se presenta claramente más débil. En efecto, constatamos fuertes variaciones interindividuales al menos en cuatro ítems: «danzar», "volcar», «explotar» y «romper». En lo que respecta al resto de los ítems (andar, jugar, avanzar, subir y prepa- 
rar), los describen, al igual que los demás grupos, de manera claramente durativa.

Las correlaciones entre niveles (Tabla V) son siempre elevadas y significativas al menos a $p<.02$. Los resultados obtenidos por los sujetos de 2. ${ }^{\circ}$ son los más débilmente correlacionados con el resto de los grupos. Así, en lo que respecta a la duratividad, las representaciones cognitivas de la mayoría de los ítems verbales parece que se establecen claramente y de forma ampliamente compartida entre los diferentes niveles. Sin embargo, algunos verbos manifiestan variaciones bastante sensibles en algunos niveles.

\subsubsection{La resultatividad}

La Tabla III evidencia una fuerte homogeneidad (intra e intergrupos) en las descripciones de algunos verbos: para la mayoría de los sujetos de los cinco grupos considerados «andar», «jugar», "danzar», «avanzar»y «subir» son descritos como no resultativos (con la excepción de "avanzar" que en $4 .^{\circ}$ es considerado como resultativo), mientras que "volcar", "explotar" $\mathrm{y}$ «romper» dan lugar a descripciones resultativas (con la excepción de «volcar» que, en $2 .^{\circ}$, produce variaciones interindividuales). Sin embargo, el verbo "preparar" se caracteriza por una ausencia de acuerdo intergrupos.

Al igual que en el caso de la dimensión de la duratividad, todas las correlaciones son significativas, al menos a $\mathrm{p}<.01$.

En resumen, la similitud de las representaciones cognitivas con respecto a la resultatividad parece, al menos, tan importante como la de la duratividad.

\subsubsection{La continuidad}

De acuerdo con los resultados de la Tabla IV, la mayor parte de los verbos presentan descripciones de continuidad en los cinco grupos. En 2. dos los ítems verbales se describen de forma continua. Esta constatación nos lleva a proponer dos preguntas que, por el momento, no nos es posible responder: ¿las representaciones cognitivas de los verbos estudiados son, por sí mismas, representaciones claramente continuas? y/o ¿la dimensión "continuidad" ha sido tenida en cuenta por los niños de este grupo?

Aunque la mayor parte de los verbos parece describir procesos continuos, observamos:

- Por una parte, la existencia de variaciones interindividuales (sobre todo en el grupo de adultos y en $4 .^{\circ}$ ).

- Por otra parte, que ciertos verbos (andar, danzar) son descritos de manera iterativa por los sujetos de más edad de nuestra muestra (adultos, $8 .^{\circ}$ y $6 .^{\circ}$ en el caso de «andar" y adultos y $80^{\circ}$ en el de «danzar».

Las correlaciones entre niveles (Tabla VII) son, también en este caso, elevadas y mayoritariamente significativas. Las más débiles se observan entre los grupos de $8 .^{\circ}$ y de $2 .^{\circ}(.606 \mathrm{~ns})$, entre los grupos de $8 .^{\circ}$ y $6 .^{\circ}$ (.734 ns) y entre los grupos de adultos y de $2 .^{\circ}(.623 \mathrm{~ns})$.

En resumen, la continuidad no constituye una dimensión discriminante. No permite diferenciar categorias de proceso - al menos con los verbos utilizados en este trabajo- en función de su carácter más o menos continuo, y no aparece como una dimensión que pueda proporcional informa- 


\section{2}

ción de eventuales modificaciones de las representaciones cognitivas en función de la edad. Hay que recordar, no obstante, que los verbos utilizados han sido seleccionados sobre la base de su duratividad y su resultatividad potenciales. Los resultados habrían sido diferentes, sin duda, si la iteratividad hubiera sido también manipulada como variable independiente.

\subsubsection{Conclusión}

De las tres dimensiones consideradas, sólo dos parecen ser discriminantes para los verbos que hemos estudiado: la duratividad y la resultatividad. Los diversos ítems son objeto de un elevado consenso en lo que respecta a las representaciones cognitivas. Así, se pueden oponer procesos percibidos claramente como resultativos o no resultativos, durativos o no durativos. Sin embargo hay algunos procesos que parecen ocupar posiciones intermedias, al existir un débil nivel de acuerdo entre los sujetos. Aunque las correlaciones entre grupos son siempre relativamente elevadas y mayoritariamente significativas, se manifiestan algunas diferencias entre los valores de ciertas dimensiones de las representaciones cognitivas, sobre todo en determinados niveles e ítems. Es, por esto, necesario un análisis complementario, más sintético.

\subsection{Análisis global}

El análisis factorial de correspondencias (AFC) que hemos utilizado tiene, para el problema que tratamos de abordar, una triple ventaja:

- Permite un tratamiento sintético de los resultados, considerando simultáneamente el conjunto de las variables.

- Revela, dentro del conjunto de las variables potenciales, las dimensiones más pertinentes.

- Evidencia, también, oposiciones y agrupamientos en función de esas dimensiones.

Resulta, pues, un análisis muy adecuado para nuestro objetivo.

En todos los grupos, el eje 1 opone las acciones resultativas no durativas a las acciones no resultativas durativas y explica, al menos, el $73 \%$ de la inercia $(.73<\%<.90)$. El eje 2 permite, bien sea oponer los procesos durativos resultativos al conjunto de los demás procesos $\left(4 .^{\circ}\right)$, o bien, hacer aparecer las variaciones interindividuales sobre las descripciones de los procesos (adultos, $8 .^{\circ}, 6 .^{\circ}$ y $2 .^{\circ}$ ).

Para cada uno de los cinco grupos considerados, las proyecciones sobre el eje 1 de los puntos que representan las modalidades de las dos variables estudiadas (DO, DN, RO, RN) y los nueve ítems verbales (Cf. Figura 5) muestran que:

- "Volcar», «explotar» y "romper» se agrupan siempre en torno a los puntos que simbolizan la resultatividad (RO) y la no duratividad (DN). Estos tres verbos son descritos de manera resultativa no durativa por todos los sujetos, excluyendo los niños de $2 .^{\circ}$; en efecto, dentro de este grupo se registran variaciones interindividuales en la variable "duratividad" (en los tres verbos), así como para la variable "resultatividad" (en "volcar»).

- En el otro extremo del eje, próximos a los puntos que simbolizan la duratividad (DO) y la no resultatividad (RN), los verbos «andar», «ju- 


\section{3}

FIGURA 5

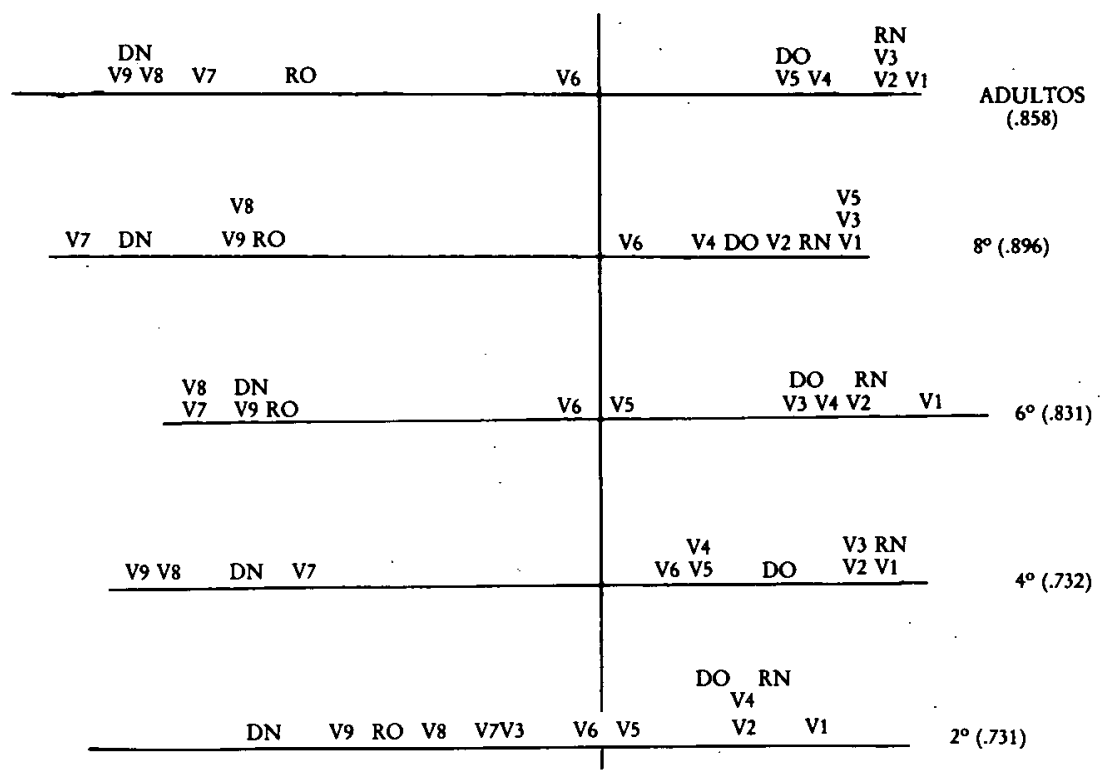

V1: ANDAR, V2: JUGAR, V3: DANZAR, V4: AVANZAR, V5: SUBIR, V6: PREPARAR, V7: VOLCAR, V8: EXPLO TAR, V9: ROMPER

DO: DURATIVO, DN: NO DURATIVO, RO: RESULTATIVO, RN: NO RESULTATIVO.

Proyección sobre el eje 1 de los verbos y de los descriptores de los procesos en función de los grupos de edad.

gar», «danzar», «avanzar» y «subir» forman un grupo relativamente homogéneo. Estos cinco verbos describen generalmente procesos no resultativos durativos. Hay que señalar, sin embargo, que en $22^{\circ}$, el acuerdo interindividual para el verbo "danzar" en lo que a la duratividad respecta, es menor que el obtenido en los demás grupos. Igualmente, podemos advertir que si "avanzar» es percibido de manera durativa en $4 .^{\circ}$, da lugar, a menudo, a una descripción resultativa (DORO). En $6 .^{\circ}$, para finalizar, obtenemos, en el caso de «subir», variaciones interindividuales para la variable duratividad.

El verbo "preparar" sufre desplazamientos en función de los grupos. Aparece claramente durativo en cuatro grupos (el grupo de $6 .^{\circ}$ representa la excepción, con variaciones intersujetos). Sin embargo, su carácter resultativo está lejos de ser comprobado; en efecto, se pueden observar fuertes variaciones intra e intergrupo en lo que respecta a la dimensión resultatividad.

\section{CONCLUSION}

El objetivo de este trabajo era plantear, en términos operativos, el problema de la estabilidad o del desarrollo de un cierto número de dimensiones (duratividad, resultatividad, continuidad) estimadas pertinentes respec- 


\section{4}

to de la representación cognitiva de los tipos de procesos. Para esto, a fin de evitar las dificultades y los riesgos de confusión ligados al recurso de la verbalización, hemos utilizado un método consistente en pedir a los sujetos que realicen una representación gráfica de las dimensiones estudiadas en nueve verbos.

Los resultados obtenidos en adultos y en niños escolarizados en $4 . .^{\circ}, 6 .^{\circ}$ y $8 .^{\circ}$ confirman la pertinencia del método, que hace aparecer una neta dicotomía que opone los procesos resultativos no durativos a los no resultativos durativos. Parece, pues, que se ha obtenido un amplio consenso en las representaciones asociadas a los nueve verbos estudiados.

Los datos recogidos en $2 .^{\circ}$, aunque no cuestionan esencialmente los hechos observados en los demás grupos, denotan un menor acuerdo interindividual, sobre todo en algunos verbos (danzar, preparar, volcar, explotar y romper). Parece que la representación cognitiva asociada a ciertos tipos de procesos no estuviera todavía completamente determinada y estabilizada.

Así, estos primeros resultados revelan que la representación cognitiva asociada a los tipos de procesos (al menos los nueve aquí estudiados) varía en función de la edad. Después de estos primeros trabajos convendría continuar con la investigación en niños más jóvenes.

En definitiva, estos resultados plantean un doble problema. En primer lugar, las diferencias de representaciones cognitivas entre sujetos de la misma edad relativas a determinados tipos de procesos (sobre todo "preparar») conducen a replantear el problema del impacto de estas representaciones sobre la selección de las formas verbales. Conviene, en efecto, investigar si los tiempos utilizados por un sujeto determinado son congruentes con la representación cognitiva del tipo de proceso considerado (por ejemplo, el imperfecto con DORN) o si, por el contrario, no hay ninguna regla que rija las asociaciones.

A continuación se plantea la cuestión del origen de las transformaciones de las representaciones cognitivas en función del desarrollo. Son posibles dos grandes categorías de hipótesis. La primera consiste en invocar una explicación en términos de extracción progresiva por el sujeto de las características objetivas del proceso considerado (habría, como para los objetos, procesos más prototípicos que otros y su representación se encontraría establecida más precozmente). La segunda considera que las características de la lengua materna - por ejemplo la concurrencia fuerte entre «explotar" y el pretérito indefinido o el pretérito perfecto, y la rara asociación de este verbo con el imperfecto- determinan (o contribuyen a) la utilización y la forma de la representación cognitiva asociada a un proceso determinado en una lengua determinada.

Son, pues, necesarias investigaciones complementarias que aborden la génesis y la comparación, interlenguas, para poder establecer el peso de estos dos tipos de influencias. 


\section{Referencias}

ANTINUCCI, F., y MiLleR, R. (1976). «How children talk about what happened . Journal of Child Language, 3, pp. 167-189.

BRONCKART, J. P. (1976). Genèse et organisation des formes verbales chez l'enfant. Bruselas. Dessart \& Mardaga.

FAyOL, M. (1983). «L'acquisition du récit: un bilan de recherches». Revue Francaise de Pédagogie, 62, pp. 65-82.

FAYOL, M.; ABDI, H., y GOMBERT, J. E. (1989). «Use of past tense inflections in French: developmental study of the interaction between type of process and context *. European $B u-$ lletin of Cognitive Psychology, 9 (3), pp. 279-295.

- (1988). "Temps et types de procès. Une étude descriptive et développementale». En N. Tersis y A. Kihm (eds.): Temps et aspects. Actes du Colloque C.N.R.S. París, 24-25 octubre 1985. París: Peeters/Sela\}, 284, pp. 239-254.

GILI GAYA, S. (1974) Estudios de lenguaje infantil. Barcelona: Biblograf.

HARNER, L. (1982). «Talking about the past and the future». En W. J. Friedman (ed.): The developmental psychology of time. Nueva York: Academic Press, pp. 141-169.

HERNÁNDEZ PINA, F. (1984). Teorias psico-sociolingüisticas y su aplicación a la adquisición del español como lengua materna, Madrid: Siglo XXI.

Malrieu, P. (1976). "L'expresion verbale de la temporalité avant 4 ans». Bulletin de Psychologie, 26, pp. 340-349.

Malrieu, P., y LATERASSe, C. (1976). "L'expresivité dans le langage enfantin». Journal de Psychologie, 3 (4), pp. 419-443.

SMITH, C. S. (1980). "The acquisition of time talk: Relations between child and adult grammar". Journal of Child Language, 7, pp. 263-278.

SZAGUN, G. (1978). *On the frequency of use of tenses in English and German's children spontaneous speech*. Child Development, 49, pp. 898-901.

Vendler, Z. (1967). Linguistics and Philosophy. Ithaca: Cornell University Press.

WEIST, R. M. (1984). "Tense and aspect: Temporal systems in child languagew. En P. Fletcher y M. Garman (eds.): Language acquisition: studies in first language development. Cambridge: Cambridge University Press.

\section{Extended summary}

To study the functioning and development of verbal forms several relevant factors were defined. One of these factors is the construction of a referent relative to the source of the utterance, which is called the deictic dimension, and defines the construction of the past-present-future axis. The speaker's viewpoint is interrelated with this factor, and it conditions the speaker's selection, for instance, between the different forms which the past might adopt. Together with the cognitive elaboration of this double referential system, it seems that exposure to an input of forms sufficiently varied and frequent is also indispensable for the acquisition of the verbal paradigme of any language. Another factor which has received relatively little attention is the cognitive representation of the characteristics of the process -or "Aktionsasrt» - of each verbal lexeme. This last factor, as several authors have pointed out, would condition the existence of a correlation, for example, between verbs expressing durative and non-resultive processes and the imperfect form.

The present study focusses on this last issue. Empirical data is provided on how the adults and children in our sample represented the type of process described by each verbal lexeme.

The verbs studied has been limited to nine (walk, play, dance, advance, go up, prepare, turn over, explode, and break), selected as a function of the three most significant factors in the representation of the type of verb 
process: durativity, resultivity and iterativity or continuity. Their influence on the selected verbs is the specific aim of the present study.

The use of graphic representation (longer or shorter lines, limited or unlimited, continous or discontinous) to «express" how subjects represent the type of process implicit in each verbal lexeme, has proved to be very appropiate, for it has helped to avoid the subjectivity and reticence which the need to verbalize and give ones opinions might provoke.

The study population were 42 History undergraduates in their fourth year -of a five year degree- at the University of the Basque Country in Vitoria, and 72 children studying grades $2,4,6$ and 8 in a public primary school in Vitoria. The first language of all subjects was Spanish.

The procedure followed in the data analyses was the following: first, percentages were calculated for the three dimension for each verb studied; second, correlations between these percentages and all the groups were obtained; finally group by group factor analyses of correspondence were undertaken. In this way, it was possible to find out, on the one hand, whether within and between groups percentages for the representations obtained for each verb are kept constant; and through factor analysis, on the other hand, whether when the three dimensions studied (durativity, resultivity, and iterativity) are considered simultaneously they showed similar regroupings of verbal items in all the groups.

The results show: a) that the method used is valid, for a clear dichotomy -occurs opposing resultative/non-durative processes to non-resultive/ durative processes; b) that the process' durativity and resultivity is represented in a very similar manner by college students and schoolchildren in the three higher age groups -average age $9,11,13$ years-, though the youngest children -average age 7 years - show lower interindividual agreement. This means that representation of the characteristics of the verb process is modified with age; c) that durative or iterative factors are not discriminat factors in the present experiment.

It is now necessary to explain further to what extent the different influence exerted by resultivity, durativity or continuity factors, here associated with each verb, is identified with specific time forms of the Spanish verbal system; and how the subjects in the course of development come to associate these factors when they occur. Further research addressing its genesis and undertaking interlinguistic comparisons are necessary in order to complete the data obtained here. 